\title{
ROBOTIC NDE INSPECTION OF ADVANCED SOLID ROCKET MOTOR CASINGS
}

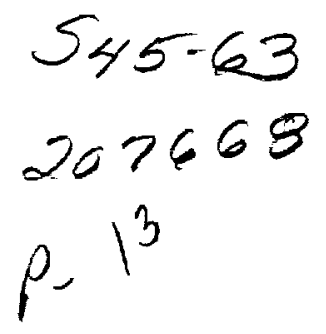

The Advanced Solid Rocket Motor program determined the need to inspect ASRM forgings and segments for potentially catastrophic defects. To minimize costs, an automated eddy current inspection system was designed and manufactured for inspection of ASRM forgings in the initial phases of production. This system utilizes custom manipulators and motion control algorithms and integrated six channel eddy current data aquisition and analysis hardware and software. Total system integration is through a personal computer based workcell controller. Segment inspection demands the use of a gantry robot for the EMAT/ET inspection system. The EMAT/ET system utilized similar mechanical compliancy and software logic to accomodate complex part geometries. EMAT provides volumetric inspection capability while eddy current is limited to surface and near surface inspection. Each aspect of the systems are applicable to other industries, such as, inspection of pressure vessels, weld inspection, and traditional ultrasonic inspection applications.

\section{Background}

Initial manufacture and subsequent refurbishment of the space shuttle Advanced Solid Rocket Motor (ASRM) demand precise inspection of the motor casings, both in the forging and segment phases of production, to preclude catastrophic failure. Robotic NDE inspection for case discontinuities was determined essential to achieve the program goal of ensuring overall case integrity. Two inspection points were identified in the ASRM cycle. The first inspection would identify surface fissures created during the forging and heat treatment manufacturing steps. Detection of flaws at this stage prevents scraping components downstream in the process. inspection of segments (assembled forgings) during initial manufacture and refurbishment is the second point, and occurs early in the production cycle to ensure new segment integrity and check for cracks propagated during splashdown.

The sizes and complexity of forgings and segments requires the use of advanced robotic techniques for automated inspection employing state-of-the-art NDE. The approach to each inspection was driven by unique functional requirements requiring different robotic and NDE methods. In both cases, Babcock \& Wilcox, CIM Systems was contracted to provide the innovative and rugged solutions.

\section{Eddy Current Inspection System}

ASRM cylindrical forgings span a wide range of sizes and complexity of features. Inner diameters range from 142.495 inches to 149.625 inches and outer diameters from 145.180 inches to 161.750 inches. Heights range from 34.50 inches to 148.420 inches. The forgings can weigh as much as 15,000 lbs. As a result of heat treatment, component outof-roundness (circularity) could be as much as seven inches. Features may be final machined or forged, requiring additional machining in subsequent processes. Complex geometries include radii, corners, chamfers, weld prep transitions, protrusions, flanges, and multi-axis curvatures. The critical flaw size was set at 0.125 inch long by 0.035 inch deep for all inspections.

A vertical five axis system comprising two, two axis manipulators and a rotary table (figure 1) controlled by an eight axis servo controller provides the necessary motion for forging inspection at the Babcock \& Wilcox, Aerospace Components Division, the manufacturer of ASRM segments. Six, single channel eddy current (ET) instruments are used for surface and near-surface flaw detection. A personal computer based, workcell controller integrates the motion control, data acquisition, and 


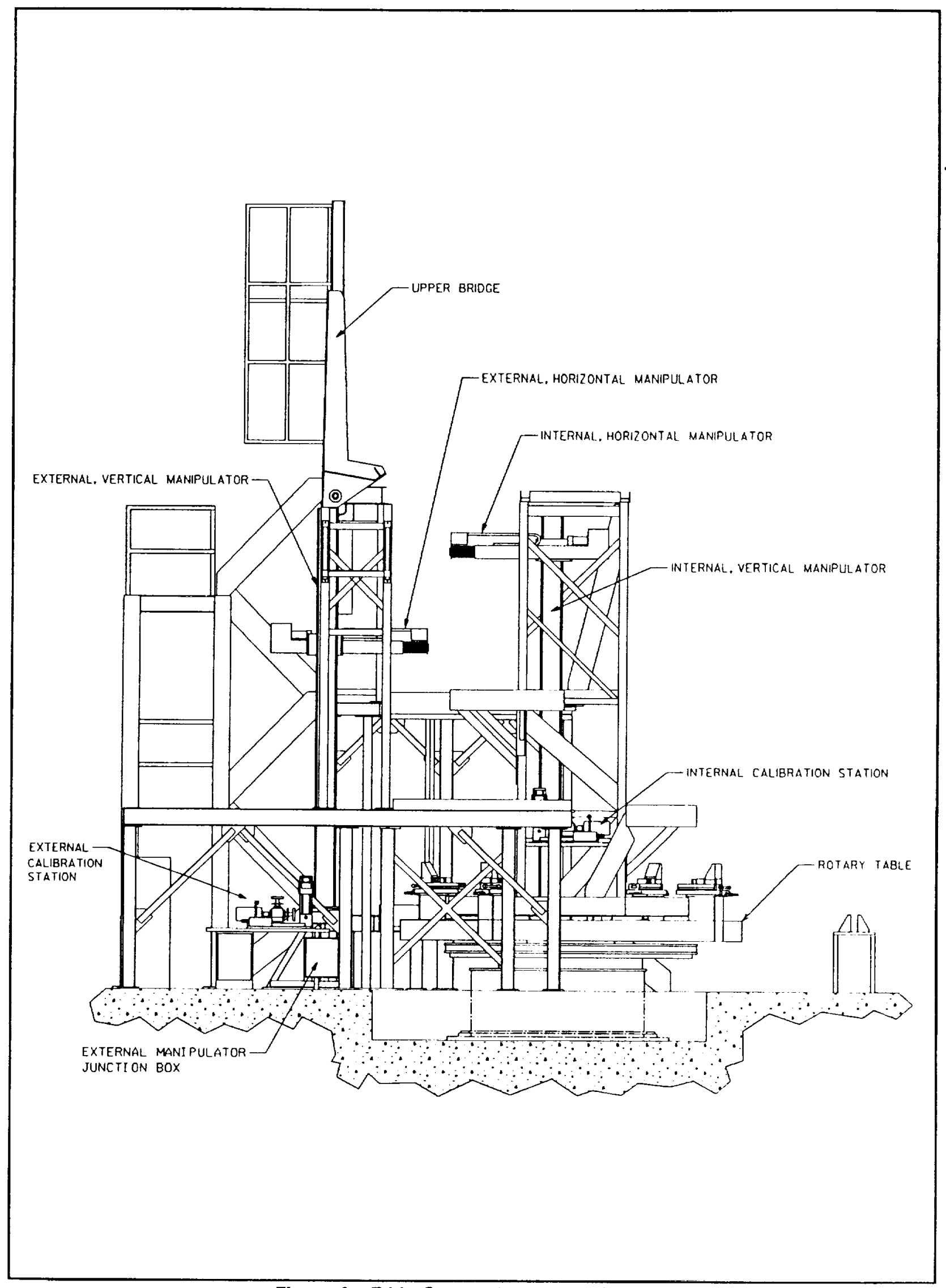

Figure 1. Eddy Current Inspection System 
horizontal axis for the positioning of over thirty pieces of unique eddy current tooling. Critical to this application is maintaining proper part contact. The unique solution incorporates four axes of compliancy: $x, z$, pitch, and yaw. Adaptive control is utilized on the $x$-axis compliancy to overcome part out-of-roundness. Sensory techniques and custom algorithms are employed on other compliancy axes to maintain part contact and detect possible collision.

\section{Manipulators and Motion Control}

\section{Horizontal Axis Manipulators}

The horizontal axis manipulators are custom designed extendable/retractable assemblies mounted on the vertical axis carriage for both the internal and external vertical axes (figure 2). Each is perpendicular to the vertical axis and mounted so that it is on a radial line of the rotary table. This is important so that the tooling mounted to the end of the horizontal axis is perpendicular to the surface of the ASRM part. Otherwise excessive vibration will occur.

The purpose of the horizontal axis is to position the end-of-arm tooling in towards or out from the surface of the ASRM part. Since ASRM parts are not perfect circles, a special subassembly is incorporated into the horizontal axis to compensate for the out-of-roundness. This subassembly is the linear, or $x$-axis, compliancy device, properly known as the compliancy device assembly (figure 3 ). This is mounted to the free end of the axis. At the end of the linear compliancy device is a flange mounting plate for mounting tooling components.

Extension and retraction of the horizontal axis is accomplished as follows. A fixed ball screw mounted concentrically within a large hollow shaft, with the ball nut secured to the rear of the shaft, allows the hollow shaft to extend and retract as the ball screw turns and the ball nut translates down the screw. Linear ball bearing bushings mounted in the forward end of the axis housing allow the manipulator to be subjected to large transverse loads without detriment. This feature, and space constraints, were the deciding factors in designing a custom horizontal axis versus using an off-the-shelf linear actuator. Transverse loads on the order of 300 pounds can be applied. Off-the-shelf actuators can typically withstand ten percent of their axial load, maximum, as a transverse load, making them inefficient for transverse load applications. Cam followers are used to eliminate any rotational movement of the hollow shaft and ball screw combination due to overhanging loads off the axis.

The fixed end of the ball screw at the rear of the axis housing is driven in parallel using a positive power transmission belt and sprockets. A servo motor fitted with an absolute encoder provides the necessary driving rotary motion and position feedback. The axis is capable of 108 inches per minute with a total stroke of 35 inches between soft limits.

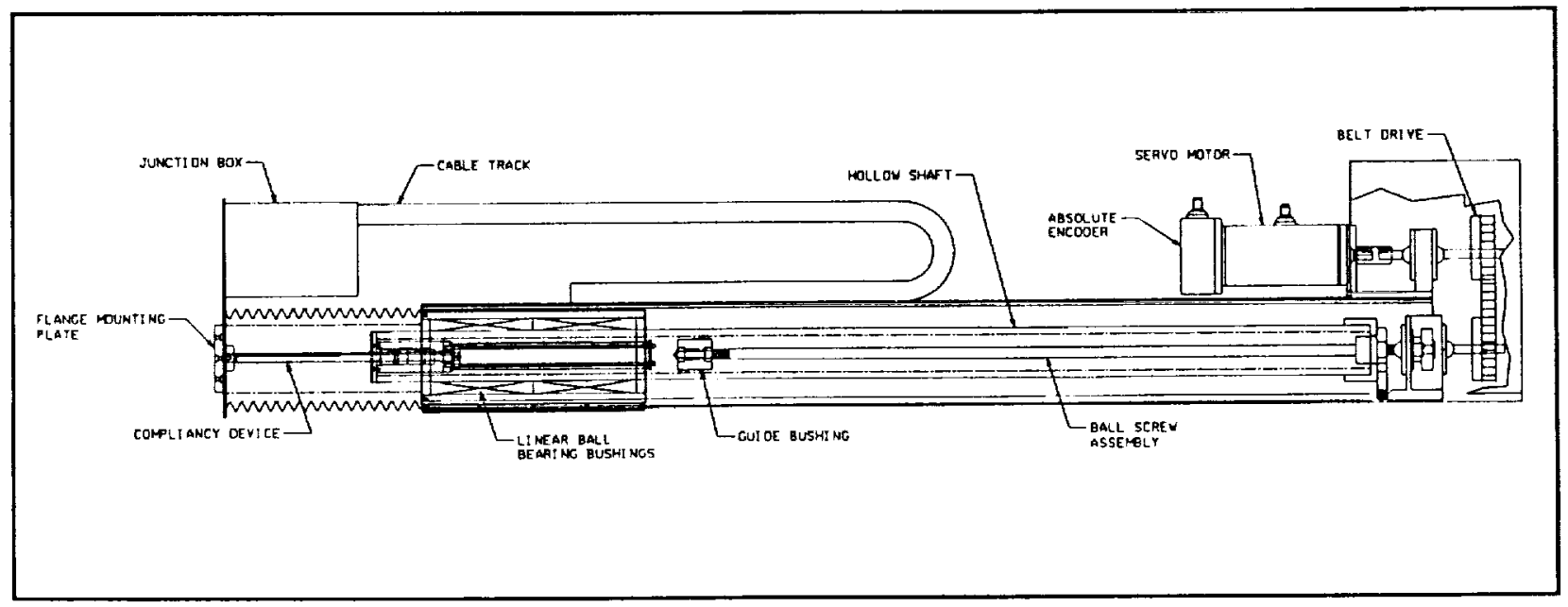

Figure 2. Horizontal Axis Manipulator. 


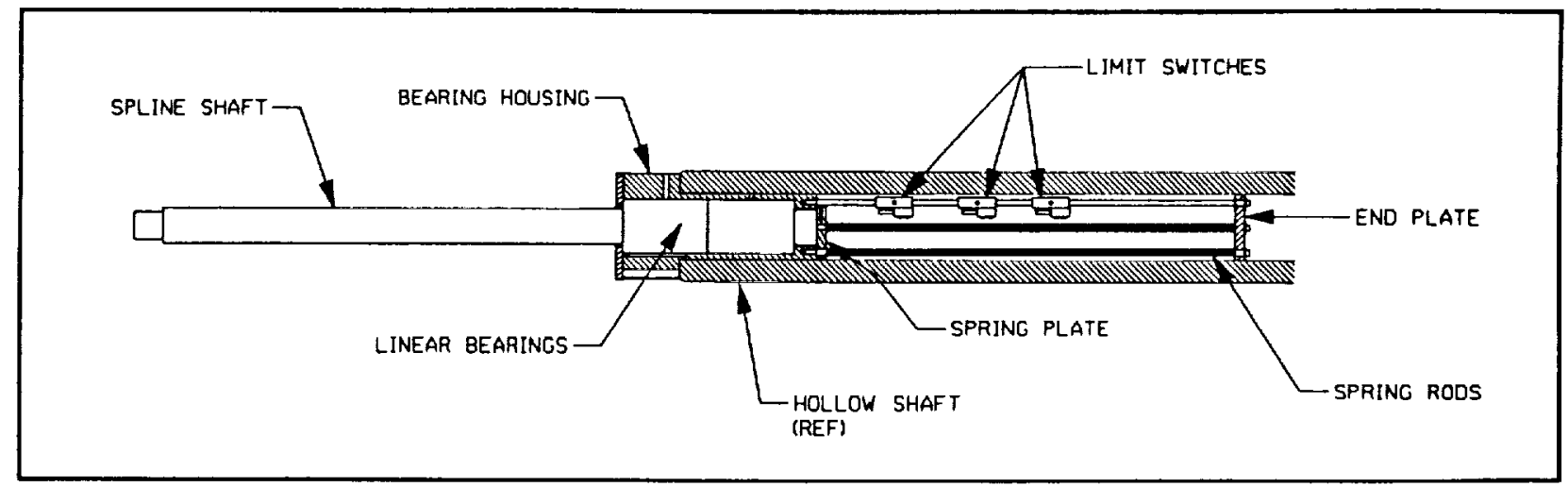

Figure 3. Compliancy Device Assembly

\section{Compliancy Device Assembly}

As stated above, this assembly is mounted into the hollow shaft in the end that extends and retracts. The design utilizes a ball spline assembly for smooth linear motion, transverse load capacity, and constraint from rotational motion. The ball spline ball bearing bushings are mounted in a housing fixed within the end of the hollow shaft. The spline shaft end within the hollow shaft is mounted to a plate and spring combination which provides sufficient spring force to push tooling and sensors against the ASRM component maintaining sensor-to-part contact. Three sensors mounted to the compliancy device assembly within the hollow shaft are used to detect the location of the end of the shaft. If the ASRM component forces the shaft inward, the rear sensor is actuated causing the motion controller to retract the horizontal axis to the center of the compliancy device. If the ASRM component moves away causing the spline shaft to extend, the forward sensor will be actuated causing the motion controller to extend the horizontal axis.

This combination of limited travel, spring resistant compliancy, sensor feedback, and control permits horizontal axis compliancy within the entire stroke of the axis, 35 inches.

\section{Arm Compliancy Device Assembly}

Though actually categorized as part of the end-of-arm tooling, the arm compliancy device assembly (figure 4)is discussed here because the item remains attached to the end of the horizontal axis for all inspections. It is mounted to the end-of-arm mounting flange. Attached to this assembly is a integrated, quick change tooling dovetail which allows for rapid tool changes, alignment, and rigidity. Where the compliancy device assembly discussed above provides $x$-axis compliancy, this device provides $z$-axis and rotational compliancy.

A linear cross roller bearing assembly is mounted vertically between the base plate and an intermediate plate. Flat air cylinders are used at each end of bearing travel to provide the necessary spring force to allow resisted vertical motion of attached tooling. Independent regulated air pressure to each cylinder permits counteracting gravitational forces and aligning the tooling.

Springs sandwiched between the intermediate plate and the final tooling mounting plate allow for pivotal compliancy about two perpendicular axes.

Embedded limit sensors on the $z$ compliancy and the two rotation compliancy axes are used to detect abnormal operational conditions such as a collision between tooling and an ASRM component.

Though these features are available as off-theshelf components, space constraints dictated a custom design. In addition to the compact design, more freedom of movement for compliancy is provided than available from offthe-shelf component vendors.

\section{Vertical Axis Manipulator}

There are two vertical axis manipulators, internal and external (figure 4). 


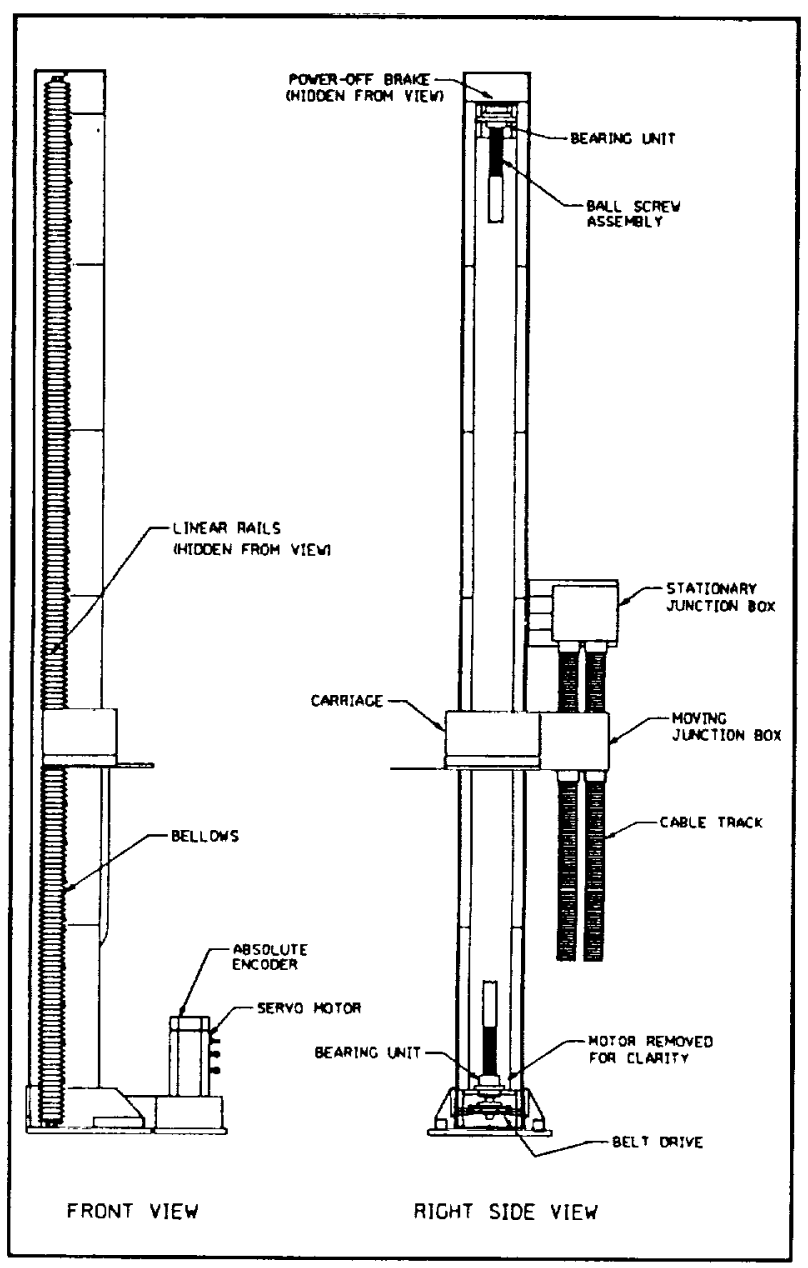

Figure 4. Vertical Manipulator Assembly

A wide flange l-beam serves as the main support structure. A linear rail fitted with two linear bearings is mounted to each flange. The vertical carriage is bolted front and back to the front and back pairs of linear bearings. The ball nut of the vertical axis ball screw is mounted rigidly to the carriage. The ball screw is mounted between the beam flanges at the top and bottom using four row angular contact bearing assemblies. A power-off $D C$ brake is spline coupled to the top of the ball screw to prevent back driving. The bottom of the screw is the drive end.

Rotation of the ball screw is through indirect 3:1 power transmission belt drive. Matched sprockets attached to the ball screw and motor shaft achieve the 3:1 gear ratio. As the ball screw is rotated, the ball nut and thus, the carriage, travel up and down the beam.

The motor is a AC brushless servo fitted with an absolute encoder for closed-loop position control. The drive allows for a maximum axis velocity of 54 inches per minute. Travel between soft limits is 157 inches.

\section{Rotary Table}

The rotary table, manufactured by Koike Aronson, Inc. , is 120 inches in diameter and capable of 0-5 RPM rotation in both directions. The center of rotation of the table (approximated as the center of the table top) defines the center of the workcell. The table is rated at 20,000 lbs capacity though is easily capable of handling 30,000 lbs.

The drive train is the geared main bearing (slew ring) driven by two pinion gears. Two gear boxes along with the gearing of the main bearing and gear ratio of the power transmission belt drive provide a 297:1 gear ratio. An DC servo motor fitted with an incremental encoder is the prime mover. A belt drive is used between the motor and input shaft of the gear boxes.

A second encoder is mounted to provide 1:1 feedback of the rotary table top position.

\section{Motion Control}

All motion of the ET Inspection system originates from the part program stored in the WorkCell controller. The program is down loaded to the motion controller via RS-232 were it is stored. The part program in association with the motion control hardware performs all closed loop motion control, 1/O control and fault handling and recovery, see system block diagram figure 5 .

The motion control system consists of five axes of motion. These axes include two (2) for the internal manipulator, two (2) for the external manipulator and one (1) for the rotary table. A sixth open loop feedback only axis is used on the rotary table for homing and position display. Each axis is controlled via a dedicated controller card located in the motion controller. The card communicates with the associated drive via an analog signal and receives feedback information from the axis encoder. 


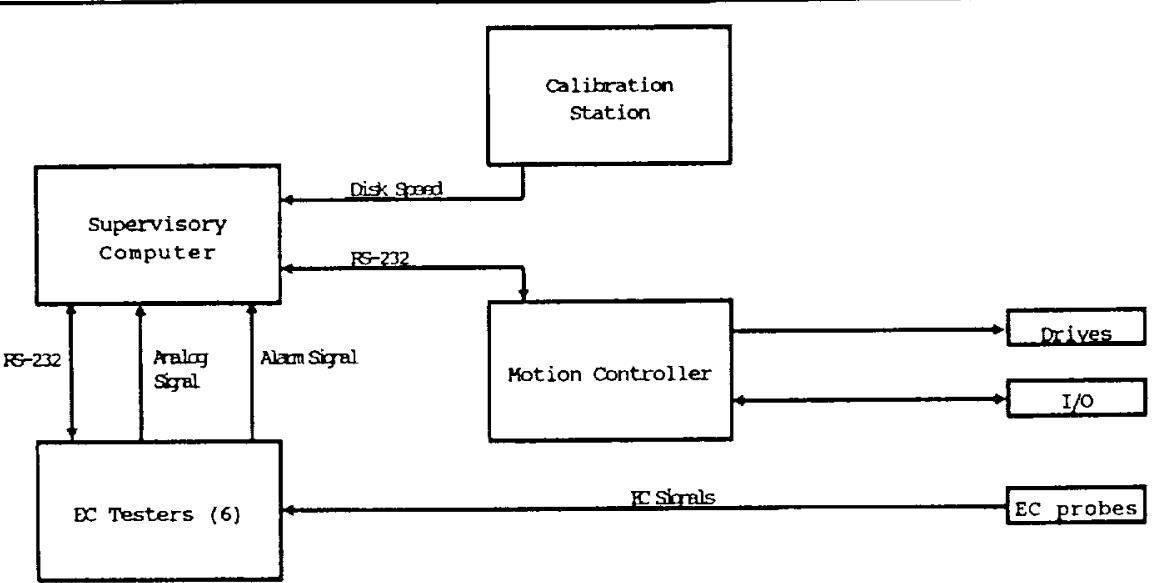

Figure 6. Eddy Current Inspection System, System Block Diagram

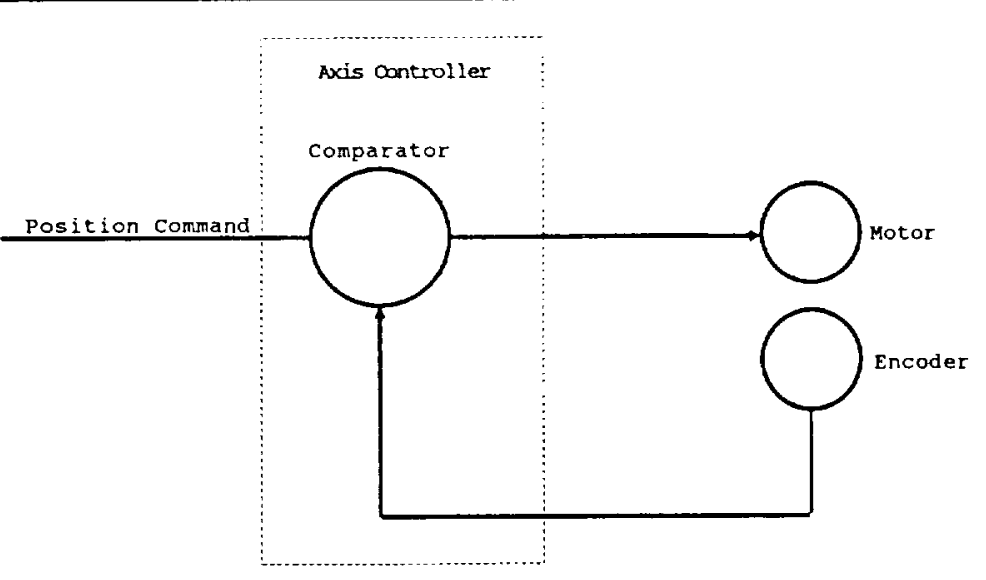

Figure 5. Feedback Loop.

Each axis of the ET Inspection system consists of a servo motor and drive amplifier. The drive accepts analog voltages from the axis controller cards, and in turn, commands the axis motor to rotate, producing axis motion.

The controller card is responsible for accepting motion commands from the motion control program and correlating this with the feedback signal (encoder) to produce an output com $f$ to the axis drive, figure 6 .

The system incorporates two types of encoders, incremental and absolute. Each manipulator axis consists of an absolute encoder with a 1024 line count. The rotary table axis utilizes two incremental encoders, one for closed loop control of the axis and the other for homing and position display functions.

\section{Tooling}

Tooling is provided to hold the eddy current probes for positioning by the manipulators. For each ASRM part feature such as a T-stiffener edge, there is a tooling setup. All setups include the tooling extension tube and end-ofarm tooling base block. The base block is used to hold the membrane eddy current probe and also serves as an attachment base for special feature tooling. Special feature tooling is provided to hold all other eddy current probes.

The base block includes two eddy current proximity probes used to detect the surface of the ASRM parts. These sense the presence of the metal surface. If the ASRM part surface is within the sensing range, it is known that the tooling wheels are contacting the part surface. The importance is that the part surface is used as a reference for positioning the tooling to 
ensure the eddy current probe is on the part surface to take data. If the part surface is not within the sensing range of the proximity sensors, the switch signal indicates a sensor liftoff fault.

\section{Sensors, Data Acquisition and Analysis}

Each of the automated NDE systems provided by CIM systems for the inspection of rocket motors consisted of Eddy Current (EC) probes. Eddy Current Test (ET) is the primary inspection method on the ET Inspection system. Due to many complex features located on the rocket motor forgings, over 30 specially designed probes and probe fixtures are needed to inspect geometries such as T-stiffeners, weld joints, chamfers and radii. Each fixture consists of a quick release mechanism to quickly provide for EC probe changes, thus a fixture may be used for many probes, see figure 7 . The probe itself not only consists of the EC coils but also provides for methods of maintaining lift-off to the inspection surface. Though many compliancy devices are provided in the system to provide for part out-of-roundness, the first two defenses for maintaining part contact are on the probe itself. A minimum of three adjustable wear pins are provided on the surface of the probe to provide a static lift-off to the rocket motor. The probe body attaches to the fixture interface plate via 3 or 4 shafts encircled with springs. The springs allow the probe to float and mimic forging movements.

Each probe consist of six (6) differential coil arrays. Each coil is staggered from the previous coil with $25 \%$ overlap to assure no flaws will pass between coils. Each coil, or channel, has a dedicated Nortec $19 \mathrm{e}$ EC tester. Each tester serially interfaces to the supervisory computer which allows for remote setting of inspection parameters such as alarm thresholds, signal gain, inspection frequencies and a variety of others. Inspection parameters are developed as a part of the calibration process. Each probe must be calibrated prior to conducting inspection of the rocket motor. The calibration consists of simulating the inspection surface

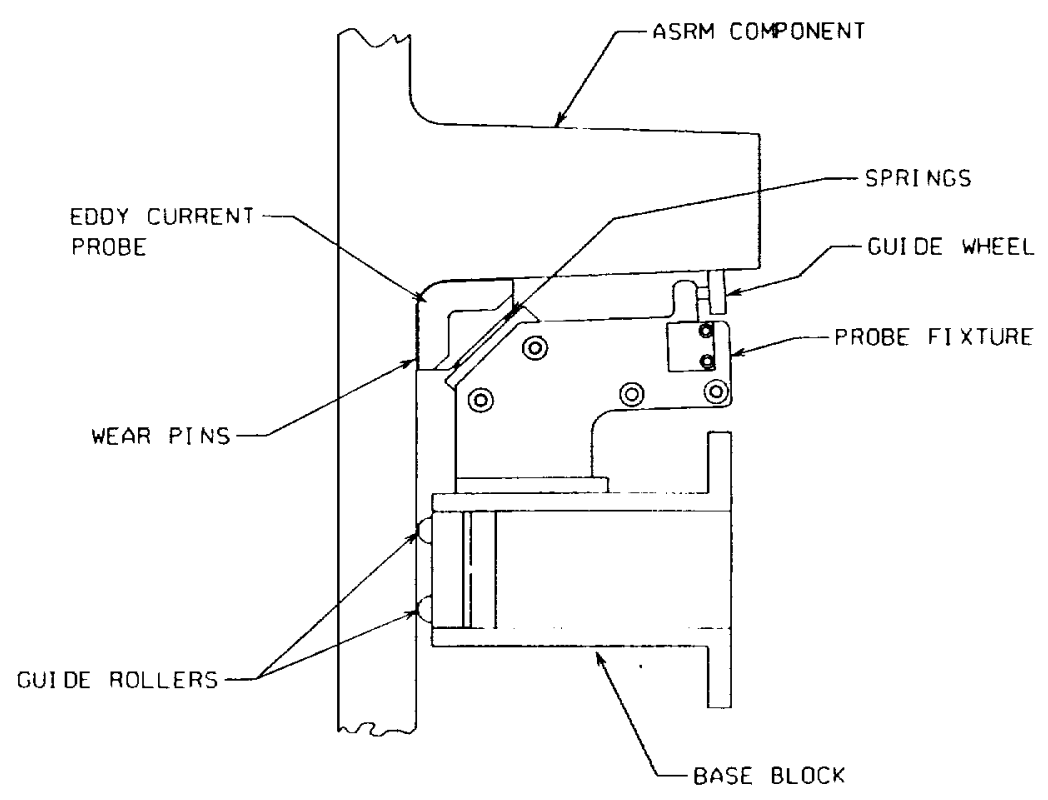

Figure 7. Eddy Current Probe, Fixture, and Base Block 
on a disk of identical material, with six (6) EDM notches of half the critical flaw size $(.125 X$ .0351 . The probe is positioned on the disk as the disk is spun at a surface speed identical to the intended inspection speed. Parameters are then adjusted on the tester to maximize the signal to noise ratio $(S / N)$ on each channel.

Data acquisition is accomplished via an analog output supplied by each of the testers. The analog signal is digitized through a Analog Devices Analog to Digital (A to D) converter card located in the PC backplane. The card digitizes each of the channels at a rate of 8.3 $\mathrm{KHz}$ with each sample point consisting of 16 bits of information. The data is buffered on the card and is retrieved on an as needed basis. The data is retrieved and stored to disk based on an alarm signal generated by the tester. The tester will generate a discrete output if the EC signal brakes the alarm threshold set during the calibration procedure. Based on the alarm signal 1 inch of data before and after the alarm is retrieved and stored to disk for analysis and archiving.

Due to the massive size of the inspection parts relative to the critical flaw size, position of the EC probe must be monitored, retrieved and stored with the inspection data upon an alarm condition. The inspection piece being a cylinder, two coordinates must be known to relocate a flaw indication, $Z$-axis of the manipulator and $\Theta$-axis of the rotary table. The rocket motor is inspected in bands and therefore position of the horizontal axis is static throughout the band. This position is relayed to the supervisory computer from the motion control subsystem and is retained for further processing in the event of an alarm condition. An encoder is used to track the position of the rotary table. Quadrature TTL level pulses are produced by a 4096 line count incremental encoder. These pulses are collected and processed by the supervisory computer.

\section{Inspection Process}

The main operator interface of the ET Inspection system is the supervisory computer. The supervisory computer provides for the development of the inspection plan or the inspection recipe. The inspection plan is developed by the NDE engineer and is a step by step sequential operation similar to $\mathrm{CNC}$ code. The plan, which can be developed off-line, is the road map for the inspection process of a forging. It provides the motion control system with motion variables, the EC testers with inspection parameters and other house keeping functions such as operators name and ID number, part identification number, probe numbers and data file names.

Key words are used to represent inspection functions. For example, the key word "EXTERNAL SCAN" and the associated parameters perform an external inspection of the forging.

EXTERNAL SCAN 5.023 .0075 .00 .5034 .00

EXTERNAL SCAN is the key word for an external inspection. The 5.0 represents the rotational speed of the rotary table in RPMs. $23.00^{\text {" }}$ is the starting $Z$ position of the external manipulator and $75.00^{\prime \prime}$ is the stopping position. The increment amount of .50" translates to 104 bands of inspection. The final parameter represents the static $X$ position of the external manipulator for the inspection. Other key words include TOOLCHANGE [position][tool ID], DOWNLOAD [file], SHUTDOWN, UPLOAD [file], MOVE [axes][coordinates] and CAL [internal or external][disk speed]. A complete inspection plan for conducting two scans may appear as follows.

CAL EXTERNAL 230

UPLOAD xyz.par

TOOLCHANGE lower $x y z$

EXTERNAL SCAN 5.0 23.00 75.00 5034.00

CAL EXTERNAL 230

CAL EXTERNAL 230

UPLOAD abc.par

TOOLCHANGE lower abc

EXTERNAL SCAN 5.0 75.00 85.00 .50 34.00

CAL EXTERNAL 230

This inspection plan first conducts a calibration of probe xyz. The disk speed of 230 RPM will translate to the same surface speed of the forging at 5 RPM. Once the calibration is complete and all EC channels respond equally with sufficient $\mathrm{S} / \mathrm{N}$ the parameters are uploaded and stored for documentation and verification purposes. The external manipulator is then positioned to the lower tool change position and the operator prompted to install tool xyz. An acknowledgement is made that the tool has been installed and the manipulator will position 
itself to the starting location of $23.00^{\mathrm{N}}$ in $\mathrm{Z}$ and $34.00^{\prime \prime}$ in $X$. The operator is then allowed to make any fine positioning if necessary and the inspection will begin. A calibration is again conducted once the scan is completed to once again verify the functionality of the $E C$ probe. The second scan is similar to the first, only at a different part of the forging. Once the inspection plan is complete a report is generated with the results of the inspection. The report includes all flaw indication locations and house keeping information.

\section{EMAT Inspection System}

Segments are multiple forgings welded end-toend. A minimum of three full size forgings, such as weld-weld or field-weld forgings are welded together. Or, for more complex segments, full size forgings are connected to multiple specialty forgings, such as T-stiffener and IETA forgings. Segments are approximately $\mathbf{4 5}$ feet high. In addition to the features encountered during forging inspection, segments have threaded and through holes requiring inspection. All features are in the final

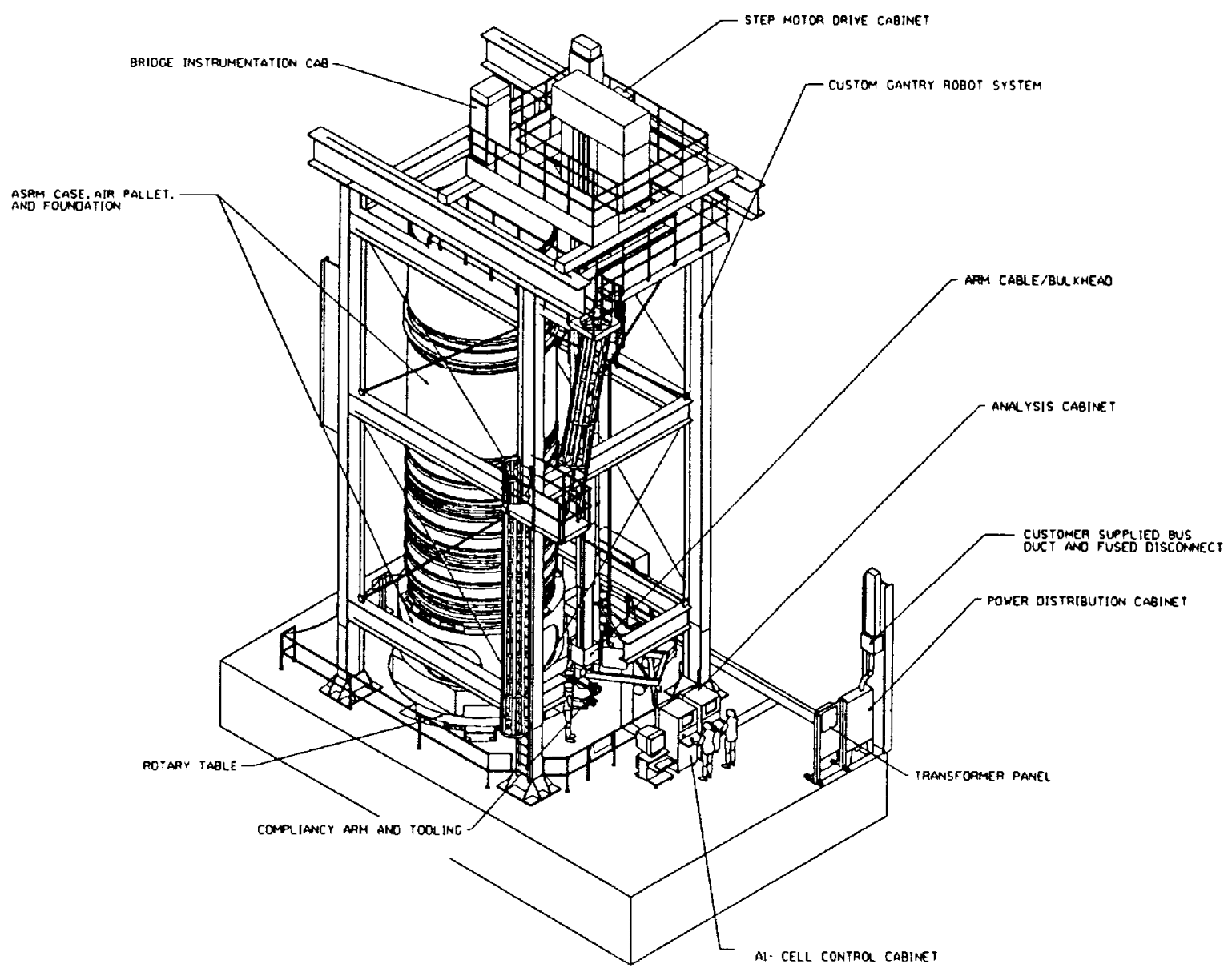

Figure 8. EMAT/ET Inspection System. 
machined state, although in the refurbishment process irregularities may be severe due to the intense heat generated during liftoff and the shock of splashdown.

Volumetric inspection of segments was specified for the assembly and refurbishment facility in luka, Mississippi prior to assembly into ASRM's. Electromagnetic acoustic transducer (EMAT) was selected as the primary NDE technique with ET being used on limited special features. EMAT is similar to UT in functionality except no couplant is required. The sound is produced by an electromagnetic acoustic interaction within the material, which facilitates high speed automated inspection. Complete, $100 \%$ inspection of a single segment takes approximately 10 hours to perform, a significant improvement over the current manual process.

In order to inspect the tall segments, a four axis gantry robot is integrated into a five axis robotic workcell (figure 8 ). Integrated through a UNIX based workcell computer are the robotic and data acquisition systems, each having a dedicated controller. Mechanical compliancy is also critical to this application and is a refinement of that used for the forging inspection station. The system features:

- A 44 foot telescoping mast

- 5 axes of coordinated motion

- CNC part programming

- Fully automated inspection techniques

- Advanced data acquisition and analysis capabilities, including $A, B$, and $C$ scans
- A multi-tasking operator interface

\section{Robot \& Control}

The Electromagnetic Acoustic Transducer/Eddy Current Test (EMAT/ET) system is responsible for inspecting new and refurbished rocket motor segments. The segments vary slightly in size and are approximately $45^{\prime}$ in height and $12^{\prime}$ in diameter. Similar to the ET Inspection system the rocket motor is placed on a rotary table and rotated while the test probes are indexed over the surface. Because of the increase in size of the inspection piece, a robotic gantry system was chosen over manipulators, see figure 8. The robot possesses an $X$ bridge assembly, a $Z$ mast assembly and a two axis wrist. The result is a 4 axis robot, $X, Z, \theta_{1}, \theta_{2}$, capable of reaching and inspecting $100 \%$ of the rocket motor. The gantry spans $65^{\prime}$ from the floor to the top of the bridge with $44^{\prime}$ of stroke on the $Z$ axis. Each axis, including the rotary table, is coordinated and controlled by the CIMROC $4000 \times$ robotic controller, see system block diagram figure 9 . The controllers functions are to perform closed loop servo control, communicate to the supervisory computer and perform system I/O.

The supervisory computer consists of a Hewlett-Packard 720 workstation running under UNIX and functions as the main operator interface and provides the platform for running the application software. A user-friendly menu system allows the operator to develop scan

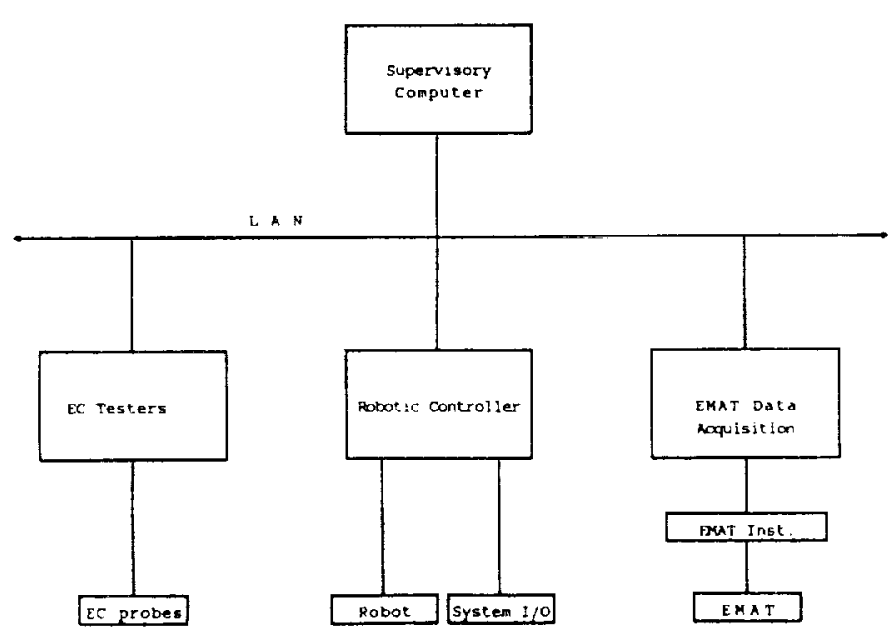

Figure 9. EMAT/ET System Block Diagram. 
plans, run inspections, download motion control functions, perform diagnostics and analyze data. An X-terminal is supplied to allow data analyzing as inspections are being run.

\section{Compliancy Arm}

A compliancy arm was designed and manufactured for use in the EMAT/ET Inspection System in order to compensate for uncertainties similar to that found with the Eddy Current Inspection System. The arm incorporates all features found in the group of compliancy features, previously discussed.

There are three sections to the compliancy arm: $X$ compliancy, $Z$ compliancy, and pitch/yaw compliancy (figure 11). Each section relies on computer adjustable, regulated air pressure to set the desired spring rate of the compliancy. This provides compensation for varying loads. Switches are used for over travel detection on the $z$ and pitch/yaw compliancy axes. The axis compliancy switches are used for adaptive control similar to the linear compliancy of the eddy current inspection system.

\section{Tooling}

Tooling for the EMAT/ET Inspection Systems consists of a dual, rotating EMAT, single, rotating EMAT, through hole EC tooling assembly, and EC probes for manual inspection. More than $90 \%$ of a segment's volume can be inspected using either the dual or single, rotating EMAT tooling assemblies.

Development of the dual, rotating EMAT was driven by the throughput requirement of 10 hours per segment, average. An EMAT probe can detect anomalies oriented within $\pm 2.5^{\circ}$ of the EMAT scanbeam. (Some tests have validated scanning for indications within $\pm 10^{\circ}$.) The scanbeam covers a forward and aft surface region that is generally trapezoidal. By using two EMAT probes mechanically coupled to rotate to the same angular

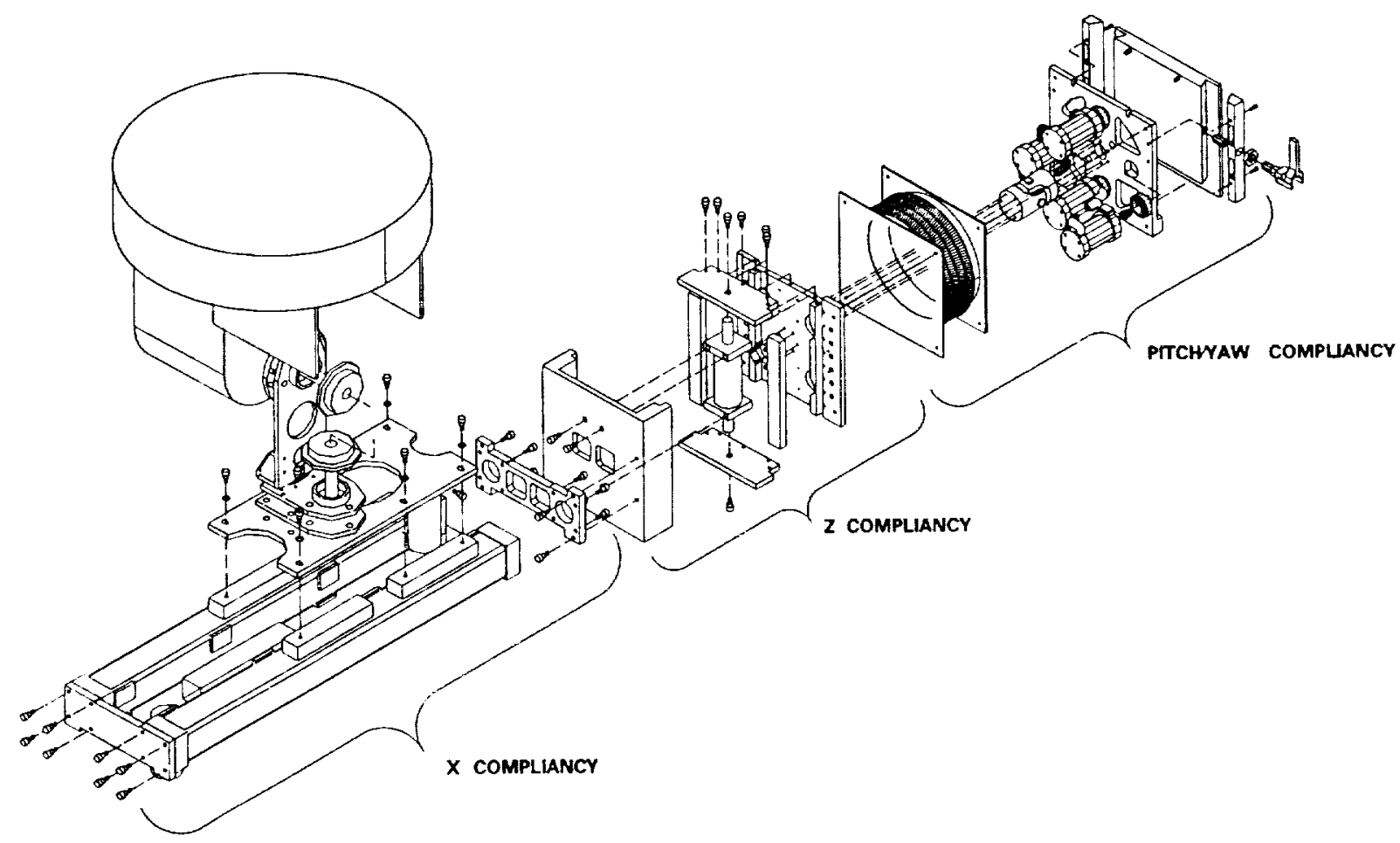

Figure 10. EMAT/ET Compliancy Arm. 
orientation and controlling overlap to cover the deadzones of the probes, an optimal scan configuration is achieved (figure 11). For each angular orientation, there is a preferred centerto-center spacing for the two probes. At lower angular orientations, the volume that can be scanned with one pass of the tooling is doubled, there is no dead zone to cover. At higher angles, approximately $150 \%$ of the volume that can be covered by one EMAT probe is scanned with the dual EMAT tooling. The greatest improvement to scanning efficiency is the minimization of increment overlap, i.e., incrementing the tooling vertically. Without the dual EMAT tooling, dead zones would be covered by increment overlap which would reduce the effectiveness of one EMAT probe to less than $50 \%$.

Step motors are used to position the dual EMAT probes both angularly and translationally, with respect to each other. Each EMAT probe is mounted with a double set of pivot points, allowing the EMAT probe to conform to the surface. Both probes are connected using a structural tube; however, the secondary probe is connected to the tube through a linear cross roller bearing which permits linear movement towards and away from the primary EMAT. A ball screw driven by one of the step motors controls the position of the secondary EMAT. A large radial bearing connects the tube to the tooling mount plate, the connection point to the robot arm. Concentric with the bearing is a spur gear driven by a mating pinion gear. The pinion gear is driven through a planetary gear box by the other step motor.

Single EMAT inspection is required in domed areas due to the complex curvature. The dual EMAT does not allow the flexibility necessary for domes because constraints between the two probes of the dual EMAT are employed to guarantee coverage between the two probes. The single EMAT tooling incorporates the rotation and translating features of the dual EMAT. Like the dual EMAT, the rotation of the single EMAT is used to adjust the probe to different orientations for randomly oriented volumetric indications. The translational feature of the single EMAT is used to scan the probe across small surfaces in lieu of using the motion capabilities of the robot. One example is scanning the vertical section of a T-stiffener ring. Step motors are used to drive the rotation and the translation of the single EMAT tooling.
A through hole, eddy current probe is fixtured in tooling to scan the many bolt holes found in the flanges of segments. The probe has one eddy current sensor coil that is spring loaded. The probe is plunged into a through hole at a known rate while rotating. This combination is necessary to ensure proper overlap to guarantee $100 \%$ coverage.

\section{Sensors, Data Acquisition and Analysis}

The EMAT/ET system not only must conduct surface inspection but also provide for a volumetric examination. Unlike ET, Ultrasonic Testing (UT) with EMATs is now coming into its own with Babcock \& Wilcox leading the way. An EMAT consists of a coil of wire and a magnet. A strong field is produced at the surface of the material by the permanent magnet. This produces an electromagnetic interaction within the material resulting in sound waves being generated. If voids such as cracks are encountered within the conductor, the wave is reflected and sensed by the receiver.

The Accusonex data acquisition system provides for data collection and analysis for the EMAT inspections. The system consists of an HP-V382 embedded controller that performs functions such as pulse control, signal digitization and serves as an interface between real-time data acquisition and data storage to disk. The system pulses the EMAT magnet and intern receives an analog signal from the EMAT instrumentation. The signal is digitized, buffered and presented to the operator in realtime in the form of A, B, or C-scans. All data, including $E T$, is stored to optical disk for analysis and archiving.

EC probes supplement the inspection in areas too small for EMAT. These areas include Tstiffener radii, bolt holes and other miscellaneous geometries. The ET instrumentation (MIZ-30) drives up to eight (8) inspection coils that are scanned over the rocket motor. The coils induce current into the rocket motor and sense changes in the electrical characteristics of the material. The electrical signal is digitized in the MIZ-30 and is transferred over a Local Area Network (LAN) to the supervisory computer for storage. The operator is presented with the data in real-time in two forms, amplitude vs. time and amplitude vs. phase. 


\section{Technology Applications}

Technologies from both systems have been implemented in other applications, particularly the robotic tooling concepts and EMAT based NDE technology. These techniques can be utilized in automated inspection of pressure vessels and other components requiring sophisticated NDE inspection to ensure part integrity.

EMAT is useful in applications requiring the output achieved from ultrasonic inspection. However, EMAT has the advantage that no couplant is required to carry the signal.

For more information, contact Glenn E. McNeelege at (804)948-1347 or Chris Sarantos at (804)948-1348. 